\title{
Validation of Kinematically Simulated Pattern HR-EBSD for Measuring Absolute Strains and Lattice Tetragonality
}

David Fullwood $^{\mathrm{a}^{*}}$, Mark Vaudin $^{\mathrm{b}}$, Craig Daniels $^{\mathrm{a}}$, Timothy Ruggles ${ }^{\mathrm{a}}$, and Stuart I. Wright ${ }^{\mathrm{c}}$

\author{
${ }^{a}$ Mechanical Engineering, Brigham Young University, Provo, UT, 84602, USA \\ ${ }^{\mathrm{b}}$ Materials Measurement Science Division, National Institute of Standards and \\ Technology (NIST), Gaithersburg, MD 20899, USA \\ 'EDAX, Draper, UT 84020, USA
}

\begin{abstract}
Cross correlation techniques applied to EBSD patterns have led to what has been termed “high-resolution EBSD” (HR-EBSD). The technique yields higher accuracy orientation and strain data which is obtained by comparing a given EBSD pattern with either a real or a simulated reference pattern. Real reference patterns are often taken from a "central" position in a given grain, where it is hoped that the material is "strainfree", and they enable the determination of relative changes in orientation and strain from that present in the lattice at the reference position. Simulated patterns, on the other hand, enable comparison of the sample lattice with that of a perfect lattice, resulting in a measure of absolute strain and orientation. However, the simulated pattern method has several drawbacks, including the need to accurately specify microscope geometry, the lower fidelity / detail of simulated patterns compared with

\footnotetext{
* Corresponding author: dfullwood@byu.edu
} 
real patterns, and the potential for microscope-specific bias in the measured patterns (such as due to optical distortion).

These drawbacks have led to much debate about the utility of the crosscorrelation technique using simulated patterns. This paper is the first to assess the accuracy of the simulated pattern method relative to the real pattern approach in a setting where accuracy can be reasonably determined, thus providing a fair assessment of the potential of the simulated pattern technique

Based upon recent developments towards a standard material for assessing strain mapping techniques, this paper assesses the overall accuracy of the simulated pattern technique. Mismatch strains are calculated using both the real and simulated pattern techniques for a SiGe film deposited on a Si substrate. While the simulated pattern technique is not as accurate or precise as the real pattern technique for providing relative strains, it provides an estimate of absolute strain that is not available via the real pattern approach.

Keywords: Cross-correlation EBSD, High resolution EBSD, Simulated EBSD pattern, Tetragonality, Validation

\section{Introduction}

Standard electron backscatter diffraction (EBSD) methods use Hough transform techniques to extract the approximate crystal lattice plane traces that are contained 
within bands on the electron backscatter diffraction pattern (EBSP) [1, 2]. The positions of the bands are analyzed to determine the angles between the planes that produce them, and these angles are subsequently analyzed to extract the orientation of the underlying crystal lattice. However, the bands on the EBSP are sections through wideangle cones of high-intensity electrons. The lines that are formed are hyperbolae; treating these as though they were straight lines, in the peak-finding algorithm that is applied in Hough space to find the centers of the bands, introduces error into the final orientation determination [3-5].

A more detailed characterization of the EBSP is possible by comparing subregions ("regions of interest") of the pattern with a reference pattern that is a projection from a known lattice onto the same phosphor, or a virtual phosphor screen. This cross-correlation technique has been commonly referred to as high-resolution EBSD (HR-EBSD; the high resolution refers to strain / orientation rather than spatial resolution) and can be divided into two fundamentally different approaches. The first approach uses real patterns taken from one region of a sample to compare with the current pattern of interest $[6,7]$. This approach results in a high quality reference pattern since it is generated in similar conditions to the pattern of interest, but details of the lattice from which the reference pattern was taken are unknown, hence the method only results in knowledge of relative distortion between the point from which the reference pattern was generated and the point from which the current pattern was emitted. The second approach uses simulated reference patterns from a known lattice $[8,9]$; thus the absolute distortion of the lattice at the scan point can potentially be 
determined. However, there are various known drawbacks with such an approach including the quality of the simulated pattern, the difficulty of obtaining accurate knowledge of the microscope geometry, and the experimental effects (such as optical distortion) that are not currently replicated by the pattern simulation algorithms . While previous publications have considered the precision of the simulated pattern approach to HR-EBSD, and its sensitivity to some of the resolution issues [9-14], this paper is the first to analyze the accuracy of the method for absolute determination of strain using a "standard" material. Stripes of SiGe film laid on a Si wafer, with well-characterized lattice distortions between the Si and SiGe $[15,16]$, are characterized using both a simulated pattern method and a real reference pattern approach.

The basic idea of HR-EBSD is to determine the distortion that must be applied to a reference structure in order to arrive at the local structure under consideration by comparing the EBSD patterns of the two structures using cross-correlation techniques [17]. Regions of interest (ROIs) are defined in the two images, and cross correlations are applied using fast Fourier transforms (FFT) to determine the shifts that must be made to the reference ROI to line up with the ROI of the image being analyzed (Fig.1). A relationship between the elastic distortion and the pattern shift seen on the phosphor, when combined with a further assumption of a traction-free surface, leads to recovery of the distortion tensor that maps the reference lattice to the lattice that is being analyzed. 
High resolution EBSD has enjoyed a string of successes in the past few years due to its ability to provide much higher accuracy relative orientation measurements than traditional Hough transform methods, along with the excellent spatial resolution of EBSD methods in general (better than $50 \mathrm{~nm}$ ). Using real reference patterns, the current attainable precision for relative orientation has been quoted to be $10^{-4}$ radians $\left(0.006^{\circ}\right)$ [7] compared with up to $0.5^{\circ}$ for Hough transform approaches (although the Hough transform precision is closer to $0.1^{\circ}$ for high quality Si patterns) $[3,4]$. Furthermore, since the full elastic distortion tensor between the reference and test patterns is recovered, the relative strain between the reference and test lattices can be determined, with precision for relative strain quoted at around $10^{-4}$. This capability has been exploited to map elastic strain gradients resulting from deformation, and consequently to determine geometrically necessary dislocation content $[8,18-20]$.

However, two significant drawbacks are associated with the real reference pattern approach. The first is that the reference pattern and the pattern of interest must have similar orientations (within about $0.5^{\circ}$ ) for the method to work well. The reference pattern is commonly taken from the center of a grain for comparison against all other points within the grain; but orientation gradients within a grain can often lead to large differences in orientation between the reference point and points at the extremes of the grain. This shortcoming has been partially resolved by a pattern remapping technique introduced by Britton and Wilkinson [11]. The second drawback can be much more detrimental to full characterization of the sample: the actual 
deformation of the reference lattice is unknown. It is often assumed that a reference point in the center of a grain is unstrained (i.e. an approximately perfect lattice), but this is generally not the case. In a recent analysis of Ti using real reference patterns, the elastic strain at the point from which the reference pattern was taken was estimated to be $0.1 \%$ [21]. The use of simulated reference patterns resolves both of the main drawbacks inherent in the real reference pattern approach, theoretically enabling the mapping of absolute strain across an entire polycrystal, rather than just the relative strain in each distinct grain. Such a map would also facilitate the resolution of so-called pseudo-symmetries for materials such as steels. Body-centered tetragonal (BCT) structures could be readily distinguished from body-centered cubic structures since the lattice elongation along the tetragonal direction would be clearly visible.

As indicated earlier, however, the move to simulated patterns also introduces different issues. The first issue relates to the fidelity of the simulated pattern compared to a real pattern. If the simulated pattern is a poor representation of a real EBSP, the correlations between the ROIs of the two patterns may be poor (see Fig. 1). Simulated patterns can be generated using a simple kinematical approach, or via a much more sophisticated (and computationally slow and expensive) dynamical algorithm. Kinematically produced patterns rely on a Bragg's law analysis to correctly position bands, but are typically simplistic, with non-realistic representations of band intensity profiles and other characteristics of real patterns. Several groups are working on libraries of patterns simulated using a dynamical approach $[22,23]$. These promise to 
provide a significant increase in accuracy of simulated pattern cross-correlation techniques. However, there is an advantage to kinematically generated patterns, in that they can be rapidly generated in an iterative fashion for a deformed lattice. EBSPs from deformed lattices can be simulated using the dynamical approach, but building a library of such patterns for all types of deformation is not currently feasible. Hence the kinematic patterns can potentially provide a reference pattern that is geometrically closer to the test pattern. The value of this advantage has not yet been tested. However, a recent comparison quantified the correlation between a real pattern and both a kinematically and a dynamically simulated pattern. Both patterns were able to distinguish a diamond pattern from patterns taken from materials with similar structure (such as silicon and magnetite). The dynamically generated patterns gave a higher level of correlation, but the kinematic patterns performed much better than expected [24]. Hence in this paper we employ kinematically simulated patterns using the approach described by Kacher [9].

The second issue with a simulated pattern approach involves the uncertainty regarding the geometry of the microscope and detector setup. The exact position of the electron emission point on the sample, relative to the phosphor screen, is termed the pattern center (PC), or the projection center. Accurate determination of this position is difficult, although there have been some recent and re-emerging advances that seem promising [10, 25-27]. Previous work indicates that an error in PC determination of 20 $\mu \mathrm{m}(\sim 0.05 \%$ of the phosphor screen for a typical system) can produce an error in 
measured strain of up to $0.06 \%$, with a reasonably linear relationship between the two [10] (see also [28] and references therein). This issue is of great importance to the results of this paper and is discussed in detail. We note that the PC issue is not generally a big concern when real reference patterns are used. The reference and scan pattern are generally taken from positions that are relatively close by on the sample, with ready and accurate adjustment for the change in PC between the two positions (assuming a well-defined planar sample surface), hence the errors in PC for the two patterns will be similar. Combining this observation with the fact that the two patterns have almost identical orientations, the PC error does not impact the calculation of relative strain significantly. Other issues of optical distortion and noise that affect the simulated pattern method are not discussed in detail, but are have minimal impact on the real reference pattern method and are implicit in the validation test itself.

\section{Method}

\subsection{Samples and Data Collection}

The samples used to test the accuracy of HR-EBSD were described in great detail in a recent study [16]. $\mathrm{Si}_{(1-x)} \mathrm{Ge}_{\mathrm{x}}$ was deposited as a blanket film, heteroepitaxially on 300 mm Si (001) wafers, using commercial processes at different compositions $(x)$. Various thicknesses of the film were applied in the range from $35 \mathrm{~nm}$ to $800 \mathrm{~nm}$ in both nominally coherent and nominally relaxed states. The coated wafer was cut into chips $25 \mathrm{~mm}$ square by NIST, and regions of the film on each chip were removed to leave stripes of film coating the Si wafer (Fig. 2). In this paper, two different wafers were chosen for inspection, based upon the previous demonstration of reasonable accuracy 
from cross-correlation techniques for those samples. The films on these samples were coherent with compositions given by $x=0.194 \pm 0.002$ and $0.279 \pm 0.003$, and with thicknesses $t=47.4 \mathrm{~nm}$ and $35.9 \mathrm{~nm}$, respectively; they will be referred to by their nominal $(t, x)$ doublet as $(50 \mathrm{~nm}, 0.2)$ and $(35 \mathrm{~nm}, 0.3)$. Composition was measured via $x$ ray photo-electron spectroscopy (XPS). X-ray diffraction (XRD) measurements were performed on the pristine blankets and processed chips and the results compared to ensure that no unintentional modification of the films had occurred during microfabrication. The films were shown to be tetragonally strained normal to the sample surface. Figure 2 shows a region of one of the samples and the coordinate system that is used.

EBSPs were collected, and initial indexing performed, using a commercial EBSD system (Oxford HKL Nordlys II, Abingdon, UK) installed on a cold tip field-emission gun scanning electron microscope (SEM) (Hitachi model 4700, Japan), typically operated at an accelerating voltage of $20 \mathrm{kV}$ with a probe current measured by Faraday cup of about $2 \mathrm{nA}$ [29]. The samples were oriented so that the images of the stripes (as seen along the 2-axis in Fig. 2) were parallel to the vertical axis of the SEM imaging screen. The electron beam was parallel to the vertical axis of the EBSD detector and $70^{\circ}$ to the 3 -axis of the sample. Line scans in the 1-direction were collected from each sample, with scans extending across several $\mathrm{Si}_{(1-x)} \mathrm{Ge}_{x}$ stripes. Typically, the scans consisted of 250 to 300 points with step sizes of $1 \mu \mathrm{m}$. EBSPs collected at each point were 1344 pixels $\times 1024$ pixels with 8 bit intensity resolution; integration times were about $1 \mathrm{~s} /$ point. The image 
processing applied to each EBSP was image division by the background and contrast optimization. The background was recorded by demagnifying the SEM image to 50x at which point the recorded diffraction background was featureless, as needed.

\subsection{Cross correlation (HR) EBSD}

The previous study using this material [16] examined the accuracy of relative strain determination using a commercially available cross correlation method (CrossCourt [30]), based upon the work of Wilkinson et al. [7]. In the current study we use a proprietary open-access code, OpenXY, that utilizes either real or kinematically simulated patterns [31]. The same algorithm for determining shifts between reference and sampled pattern is used for both real and simulated reference patterns; however, when simulated reference patterns are used, their structure is updated over several iterations to arrive at a reference pattern that is as close as possible to the sampled pattern, as described further below.

When applying the real reference pattern approach, a reference pattern was selected at the beginning of each line scan, in a bare Si region away from the $\operatorname{Si}_{(1-x)} G_{e}$ stripes; it was typically one of the first five patterns at the start of the scan. The algorithm used to determine the relative shifts of sub-patterns within the 35 evenly distributed ROIs, and the subsequent determination of relative deformation between the patterns, is described in [9]. Note that the shift that is due to the difference in position of the reference and sample points must be subtracted from the measured 
shifts. The underlying equations are similar to those used by the CrossCourt software tested in [16], as described in [7]. Landon / Kacher removed a small approximation from the equations, resulting in a slightly different set of equations [17]; however this has a smaller effect than the method of applying the traction-free boundary conditions in the software when tested on simulated patterns of randomly deformed lattices. The traction-free boundary conditions are required to enable determination of the hydrostatic components of lattice distortion, which cannot be resolved by considering only the pattern shifts in the cross correlation method.

It should be noted that when measuring lattice distortion between the $\mathrm{Si}$ substrate and the epitaxial SiGe stripes, the cross-correlation method measures the distortion as a strain. Furthermore, since it assumes that the surface of the SiGe is traction-free, it must interpret the lattice extension perpendicular to the surface as a combination of an extension in the 3-direction and compression in the 1 and 2directions (i.e. the tetragonal SiGe lattice is interpreted as a cubic lattice under triaxial strain). Thus the measured values of strain are less meaningful than the difference between the normal strains; in our case these are approximately the principal strains, with the difference between them giving the tetragonality. Note also that dilation, and therefore the zero traction assumption, does not affect our measure of tetragonality. This issue is discussed in [16]. 
If the traction free condition is applied by only requiring that $\sigma_{33}=0$ (e.g. [32]), then the error obtained when comparing (simulated) traction-free patterns of random deformations is approximately $10 \%$ higher than when the full traction-free boundary conditions, $\sigma_{i 3}=0, i=1,2,3$, are applied (see a full discussion in [33]). Hence the full boundary conditions are applied in this study by combining these three equations with the lattice deformation equations obtained for each ROI to form the full set of equations for the deformation gradient tensor. The shift between measured and reference patterns must be adjusted to compensate for the movement of the electron beam (and therefore the pattern center) between the reference and the test point. In the SEM used for these tests, one pixel of the phosphor equates to approximately $25 \mu \mathrm{m}$ of beam motion along the 1-axis. This shift due to the change in pattern center must be subtracted before the distortion can be calculated. This is illustrated in Figs. 4-7; if the shift were not subtracted, changing the beam position would cause systematic changes in the measured strain or tetragonality.

For the simulated pattern approach, a flow chart illustrating the underlying algorithm is shown in Fig. 3. The first step is to determine the pattern center for the real pattern that is being analyzed. The commercial HKL software provides a measurement of pattern center (PC) for the scan based upon a best fit approach for the measured vs theoretical band positions [10]. The PC is given by the three variables, $x^{*}, y^{*}$ and $z^{*}$, given as a fraction of the EBSP size (a fraction of the width for $x^{*}$ and $z^{*}$, and the height for $\mathrm{y}^{*}$ ) with $\mathrm{x}$ and $\mathrm{y}$ values measured from the bottom left corner of the EBSP. For 
example, the commercial software gave values for the $(50 \mathrm{~nm}, 0.2)$ scan of: 0.4921 ,

0.7430 and 0.6357 , respectively (once adjusted for the square EBSP used in the OpenXY software). When this value of PC was used for the first point in the scan, the resultant calculated strain for the presumably strain-free silicon wafer was $0.6 \%$. Various methods were attempted to try and improve the estimate of PC; however, the simplest and most accurate approach involved an optimization routine to minimize predicted strain for a trial PC. Starting from the PC given by the commercial software, a strain-free simulated pattern was produced and compared with the measured pattern. The resultant distortion between the patterns was quantified, and the PC was allowed to vary until this distortion was minimized. The optimized PC was then used by OpenXY to analyze the patterns from other scan points; note that the PC was adjusted for each point to account for beam motion across the scan. For the scan mentioned above, the optimized PC was given by: $0.5029,0.7377,0.6407$, resulting in a maximum calculated strain of around $0.08 \%$ for the silicon. The difference between the commercial and optimized value of $z^{*}$ is 0.005 , which was estimated above to give a strain error of $0.6 \%$, confirming that we are looking for PC accuracy of better than 0.001 (fraction of the phosphor screen - i.e. about $20 \mu \mathrm{m}$ ) to give a strain error of approximately $0.1 \%$ (this also indicates the accuracy that must be achieved in the PC correction for the real pattern method, discussed above). For the scans used in this study, the softwareprovided PC was typically offset from the final optimized PC by approximately $2 \%$ of the phosphor screen size (i.e. 0.02). For multiple runs of the PC optimization algorithm, the average distance of the different PCs from the mean PC (after removing one outlier) was 
0.0012 , indicating that the final PC error may be in the region of the desired accuracy of 0.001 , but it is unlikely to consistently achieve or improve upon it using this approach.

As illustrated in Fig. 3, once the PC has been determined for scan point, $k$, an initial estimate of the lattice orientation, $g^{k}$, is taken from the commercial software output, based upon the Hough transform approach. A strain-free kinematically simulated pattern is produced at this orientation, ${ }^{s} \mathrm{P}^{k}$, based upon Bragg's law. Details are given in [9]; lattice planes with the highest 8-10 structure factors are included in the simulation. The pattern is filtered (using Gaussian smoothing via a fast Fourier transform approach) to produce a profile across the bands that is closer to that of a real pattern (see Fig. 1). The filtering parameters were chosen by a design of experiments across a range of possible filter / profiling options, and are the default values in the OpenXY software.

ROIs are chosen at the same location on the simulated reference pattern and real unknown patterns, and the shifts, $q_{i}$, necessary to align these sub-patterns are calculated, in the same way as for the real reference pattern approach. These shifts then feed into the equations for calculating the lattice distortion that needs to be applied to the strain-free lattice to match the real lattice. A new simulated pattern is then created with the estimated distortion, and the ROls in this pattern are again compared with those of the real pattern (with more details shown in Fig. 3). This is repeated several 
times to arrive at the best match between simulated and real patterns, and the total distortion from the strain-free lattice is recorded.

The biggest reason for the iterative approach is the inaccuracy in the original (Hough-based) orientation measurement $\left(\mathrm{g}_{1}^{\mathrm{k}}\right)$ from the EBSD software. For the scans recorded below the point-point misorientation across the scan had a mean of $0.31^{\circ}$, with a standard deviation of $0.16^{\circ}$. The first iteration to correct rotation error has by far the largest effect on accuracy, but several (usually 3 or more) iterations beyond the initial correction result in the best precision.

\subsection{Tetragonality}

When a cubic lattice is slightly elongated (such as is the case with the SiGe in this study, or BCT steel phases that can range from zero to several percent tetragonality), traditional EBSD software has difficulty distinguishing the pattern from that generated by a cubic lattice. Some progress has been made in terms of resolving the resultant ambiguity; for example, it may be possible to identify the axis of elongation, but not necessarily the extent of the tetragonality [34]. This particular measure of absolute strain is also not accessible to HR-EBSD using real reference patterns for a typical polycrystalline material. Since a reference pattern must be taken from within the grain of interest, the relative strain of a ferrite grain cannot be compared with that of a neighboring martensite grain using this method (see $[16,35]$ for analyses on single crystals, and promising results from a lamellar material). Hence, a potentially important 
application of the simulated pattern method is to identify lattice distortion associated with lattice tetragonality across a polycrystal (for example, to identify martensite vs ferrite, along with the carbon content, which is related to tetragonal distortion).

Tetragonality for a given lattice is given by:

$$
\varepsilon^{\text {tet }}=\varepsilon_{33}^{\text {crystal }}-\left(\varepsilon_{11}^{\text {crystal }}+\varepsilon_{22}^{\text {crystal }}\right) / 2
$$

where $\varepsilon_{33}^{\text {crystal }}$ is the strain in the direction of of the c-axis. In the case of the sample materials considered here, this is normal to the chip surface in the 3 -axis direction in the $\mathrm{Si}_{(1-x)} \mathrm{Ge}_{x}$ stripes. For other materials, this direction would be determined from the strain tensor, and the equation would be adjusted accordingly.

\section{Results and Discussion}

\subsection{Strain Measurements}

Figure 4 plots the relative strain across two SiGe stripes for the two NIST chips determined using OpenXY with a real reference pattern from the Si area of the sample; i.e. the plot identifies tetragonal strain in the SiGe, calculated as a strain relative to that of the $\mathrm{Si}$. These results agree well with the predicted and measured tetragonal strains given in reference [16]. Since the tetragonal strain rather than individual values of strain were given in [16], we quantify the error in tetragonality below, rather than the error in actual strain values. 
Figure 5 plots the absolute strains across the wafers as determined using simulated reference patterns. As can be seen, the strain measurements are significantly noisier than those from the real reference pattern; for the approximately zero-strain silicon, the average absolute strain of the three principle strain components (calculated over the first Si segment for the two samples) is $0.13 \%$ with a standard deviation of $0.13 \%$ (in contrast, much higher precision is achieved using the real pattern approach in Fig. 4, as indicated by a standard deviation across the three normal strains of $0.018 \%$ ). As described above, the iterative method of finding the lattice distortion does not result in a smooth convergence, resulting in noise in the measurements. This is exacerbated by the significant error in the original orientation estimate from the EBSD software. Given that the method finds solutions that fluctuate about a reasonably constant value, an improved convergence method (perhaps with some damping) could probably reduce the noise.

We also note that in practical applications, the optimal approach is to use the simulated pattern method to estimate absolute strain at one point within a grain, and then use the real pattern from that point as the reference pattern to determine the relative strain for other points in the grain. Combining the relative plus absolute deformations will provide the overall absolute strain for all points in the grain with much higher precision than using simulated patterns for each point. 
Furthermore, for these trials the estimated PC is still not perfect, as can be seen from the fact that the average strain in the silicon is non-zero. Nevertheless, the average tetragonal strains in the SiGe bands are only around $10 \%$ different from the real pattern measurements and from the predicted values in reference [16]. These values relied upon analytical analysis based upon simple XRD, composition and assumptions of epitaxy; they have since been corroborated using improved XRD measurements that have confirmed epitaxy.

\subsection{Tetragonality Measurements}

Figure 6 plots the tetragonality determined by the real pattern method, as calculated using Eq. 1. Once again, the values for the second sample are more accurate than those for the first sample.

Figure 7 plots the tetragonality as calculated using the simulated pattern method. Table 1 summarizes the results of the measurements of tetragonality using both the real and simulated patterns. For the measured results, mean and standard deviation are taken over the first SiGe segment of the scan. The tetragonality is calculated relative to the average value of the first Si segment in accordance with Eq. 14 of reference [16]. For this exercise the standard settings on the HR-EBSD software (OpenXY) were used, with no optimization of adjustable variables. However, it is known that the results from the cross correlation approach can be sensitive to both the software parameters and to the hardware used to capture the images (e.g. data 
gathered using a JEOL microscope resulted in significantly lower estimates of tetragonality for the two samples, using both software packages). The NIST team is currently studying this variability.

\subsection{Implications of Pattern Center Error}

The results reported above emphasize the importance of accurate PC determination when using the simulated pattern approach. As discussed above, a small error in the determination of PC can lead to a large error in measured absolute strain using the simulated pattern method. The resultant error in absolute strain is highly dependent upon the orientation of the lattice; hence, if two sample points have similar orientations but different levels of elastic strain, the measured value of relative strain between the two points is fairly insensitive to PC error. Thus, as for the real reference pattern approach, the simulated pattern method could be used to determine relative strain or tetragonality even if the PC error were significant.

A further implication of PC error for the simulated pattern method is that it introduces extra noise into the iteration routine - either due to the fact that it produces a poorer starting point for the iteration, or because the PC error results in simulated patterns that could never accurately align with the real pattern. Since the simulated pattern method is particularly important for determining lattice character in polycrystalline materials (such as tetragonality, c/a ratio or grain boundary character 
across multiple distinct grains), it is important that any PC determination method also work for these materials.

It might be assumed that the strain minimization method used to determine the correct pattern center for the Si material in this paper would not work well for scan points that are not strain-free in polycrystalline samples. In fact, this is the case if only a single point in the sample is considered; however, if multiple points are used, a much more accurate PC is obtained. In order to test the potential accuracy for such an approach in these materials, patterns were simulated for 20 grains of different orientations, and with $0.5 \%$ elastic strain. The strain minimization algorithm was used to determine the PC of each of the grains - resulting in PC errors up to $4 \%$ of the phosphor size for individual grains. However, the averaged optimized PC from all the grains was approximately $0.1 \%$ (of the phosphor screen size) different from the correct value. This indicates that if similar strain levels are present across the grains of a polycrystal, the strain minimization route to determining PC may be adequate at the aggregate level.

Partial confirmation of this approach was achieved by examining the tetragonality of a TiAl sample. The expected tetragonality of the material, based upon composition, was approximately $1.8 \%$. When the PC was determined by minimizing strain on a single point of the material, the resultant tetragonality map ranged from $0.5 \%$ to $2.5 \%$, with sharp contrasts in tetragonality level between neighboring grains. However, when strain minimization was applied to 35 points in different grains, the 
subsequent PC resulted in a remarkably consistent tetragonality map, with an average value of around $1.4 \%$.

\section{Conclusions}

Tests using a proposed "standard" material for HR-EBSD validation confirmed that the use of the software package OpenXY with real reference patterns results in satisfactory results for relative strain and relative tetragonality across Si samples coated with $35 \mathrm{~nm}$ and $50 \mathrm{~nm}$ thick, $50 \mu \mathrm{m}$ wide stripes of epitaxial $\mathrm{Si}_{1-\mathrm{x}} \mathrm{Ge}_{\mathrm{x}}$. There was less than $2 \%$ error for one sample, and around $10 \%$ error for the other. The absolute error in principal strain components is not entirely meaningful, as discussed above, but can be assumed to be of similar order to that of the tetragonality. However, the precision of this measurement can be compared with quoted resolution; the average standard deviation across the principal strains $\left(1.8 \times 10^{-4}\right)$ is close to the quoted resolution limit $\left(10^{-4}\right)$. It was noticed in unreported tests that data taken from two different microscopes on the same sample resulted in significantly different absolute values of tetragonality/strain. While tuning of ROI parameters, etc. could lead to accuracy close to quoted values, the lack of a substantiated method of changing parameters for a given set of data-capture conditions prevented the authors from attempting any parameter tuning for the results given in this paper. This issue may be resolved after a planned set of tests across various data-capture environments.

Utilizing kinematically simulated reference patterns leads to noisier results; while the accuracy is still reasonable (close to $10 \%$ error for the mean tetragonality), the 
precision is 3 to 5 times worse than that of the real pattern approach. The noise is due to a combination of factors, including inaccuracy in the PC, the low integrity of the simulated patterns, along with such factors as optical distortion in the real pattern that is not replicated in the simulated pattern. These errors are exacerbated by the iterative approach used, and a more efficient convergence routine may mitigate this issue.

Higher quality dynamically simulated patterns are currently being produced by different groups, and may provide lower noise (although they currently have the disadvantage of not incorporating lattice distortion into the simulated pattern in the near term). Optical distortion could no doubt be included in the simulated pattern approach if it were characterized for a given microscope setup and fed into the simulated pattern algorithm.

While one of the largest contributors to error in the simulated pattern method is the error in the determination of the system PC, various groups are working on PC determination techniques. In the meantime, a strain-minimization approach that optimizes PC based upon the assumption of zero strain at the scan point results in a good approximation to PC for the Si. For polycrystalline samples with no zero-strain points or zero-strain points that are difficult to locate, it has been shown that if the strain minimization approach is applied to a collection of grains of random orientations, and with similar strains, the resultant PC estimate error will also be good. 
Overall the results imply that the simulated pattern method (with kinematically simulated patterns), using current techniques, can be applied to indicate absolute strain or tetragonality levels in cases where the strain / tetragonality is greater than about $2 \times 10^{-3}$.

\section{Acknowledgements}

The BYU team was supported by Department of Energy grant DE-SC0012587;

Craig Daniels was supported by an REU grant under National Science Foundation award CMMI 1404771.

\section{References}

[1] Wright SI. Journal of Computer-Assisted Microscopy 1993;5:207.

[2] Schwartz AJ, Kumar M, Adams BL, Field DP, editors. Electron backscatter diffraction in materials science 2nd Ed. New York: Springer, 2009.

[3] Wright S, Nowell M. Adv Mater Process 2008;166:29.

[4] Wright S, Nowell M, Basinger J. Microscopy \& Microanalysis 2011;17:406.

[5] Ram F, Zaefferer S, Raabe D. Journal of Applied Crystallography In Review.

[6] Troost KZ, Sluis Pvd, Gravesteijn DJ. Appl. Phys. Lett. 1993;62:1110.

[7] Wilkinson AJ, Meaden G, Dingley DJ. Ultramicroscopy 2006;106:307.

[8] Landon CD, Adams B, Kacher J. J. Eng. Mater. Technol. 2008;130:021004.

[9] Kacher J, Landon C, Adams BL, Fullwood D. Ultramicroscopy 2009;109:1148. 
[10] Basinger J, Fullwood D, Kacher J, Adams B. Microscopy and Microanalysis 2011;17:330.

[11] Britton TB, Wilkinson AJ. Ultramicroscopy 2012;114:82.

[12] Britton TB, C.Maurice, R.Fortunier, J.H.Driver, A.P.Day, G.Meaden, D.J.Dingley, Mingard K, A.J.Wilkinson. Ultramicroscopy 2010;110:1443.

[13] Maurice C, Fortunier R, Driver J, Day A, Mingard K, Meaden G. Ultramicroscopy 2010;110:758.

[14] Kacher J, Basinger J, Adams BL, Fullwood DT. Ultramicroscopy 2010;110:760.

[15] Villert S, Maurice C, Wyon C, Fortunier R. Journal of Microscopy 2009;233:290.

[16] Vaudin MD, Osborn WA, Friedman LH, Gorham JM, Vartanian V, Cook RF. Ultramicroscopy 2015;148:94.

[17] Fullwood D, Adams B, Basinger J, Ruggles T, Khosravani A, Sorensen C, Kacher J. Microstructure Detail Extraction via EBSD: An Overview. In: Barrabas R, Ice GE, editors. Strains and dislocation gradients from diffraction. Imperial College Press, 2014.

[18] Gardner CJ, Adams BL, Basinger J, Fullwood DT. International Journal of Plasticity 2010;26:1234.

[19] Wilkinson AJ, Randman D. Philosophical Magazine 2010;90:1159.

[20] Ruggles T, Fullwood D. Ultramicroscopy 2013;133:8.

[21] Britton TB, Wilkinson AJ. Acta Materialia 2012;60:5773.

[22] Winkelmann A. Journal of Microscopy 2010;239:32.

[23] Chen YH, S.U. P, Wei D, Newstadt G, Jackson M, Simmons JP, Graef MD, Hero AO. Microsc. MicroAnal. under review. 
[24] Winkelmann A, Nolze G, Vos M, Salvat-Pujol F, Werner W. Physics-based Simulation Models for EBSD: Advances and Challenges. http://arxiv.org/abs/1505.07982, 2015.

[25] Maurice C, Dzieciol K, Fortunier R. Ultramicroscopy 2011;111:140.

[26] Mingard KP, Day AP, Quested PN. Materials Science and Engineering 2014;55:012011.

[27] Biggin S, Dingley DJ. J. Appl. Crystallogr. 1977;10:376.

[28] Alkorta J. Ultramicroscopy 2013;131:33.

[29] Certain commercial equipment, instruments, or materials are identified in this paper to foster understanding. Such identification does not imply recommendation or endorsement by the National Institute of Standards and Technology, nor does it imply that the materials or equipment identified are necessarily the best available for the purpose.

[30] BLG Productions Ltd. CrossCourt. Bristol, UK, 2011.

[31] BYU. OpenXY. github.com, 2015.

[32] Wilkinson AJ, Clarke EE, Britton TB, Littlewood P, P.S.Karamched. J. Strain Anal. Eng. 2010;45:365.

[33] Hardin TJ, Ruggles TJ, Koch DP, Niezgoda SR, Fullwood DT, Homer ER. Journal of Microscopy In Review.

[34] Zambaldi C, Zaefferer S, Wright SI. Journal of Applied Crystallography 2009;42:1092.

[35] Howell JA, Vaudin MD, Cook RF. J. Mater. Sci. 2014;49:2213. 


\section{CAPTIONS AND TABLE}

Fig. 1. Region of interest (ROI) highlighted in a reference-simulated pattern (left), and real EBSD pattern with the corresponding ROI marked for comparison via cross correlation.

Fig. 2. SEM image of the end of five $50 \mu \mathrm{m}$ wide $50 \mathrm{~nm}$ thick Si1xGex stripes on a Si substrate. The coordinate system for strain measurement is indicated.

Fig. 3. Flow chart of algorithm to determine deformation gradient of lattice at scan point using simulated reference pattern

Fig. 4. Relative strain across chips (a) $(50 \mathrm{~nm}, 0.2)$ and (b) $(35 \mathrm{~nm}, 0.3)$ using HR-EBSD and a real reference pattern from the Si region of the sample $\left(\varepsilon_{11}\right.$ is red, $\varepsilon_{22}$ is green, and $\varepsilon_{33}$ is blue in the color online version). The tetragonal strains given in [16] are indicated by dashed lines .

Fig. 5. Absolute strain across chip (a) $(50 \mathrm{~nm}, 0.2)$ and (b) $(35 \mathrm{~nm}, 0.3)$ using HR-EBSD and simulated reference patterns ( $\varepsilon_{11}$ is red, $\varepsilon_{22}$ is green, and $\varepsilon_{33}$ is blue in the color online version). The tetragonal strains from [19] are indicated by dashed lines. 
Fig. 6. Tetragonality across chip (a) $(50 \mathrm{~nm}, 0.2)$ and (b) $(35 \mathrm{~nm}, 0.3)$ using HR-EBSD and a real reference pattern. The tetragonalities measured in [16] are indicated by dashed lines.

Fig. 7. Tetragonality across chip (a) $(50 \mathrm{~nm}, 0.2)$ and (b) $(35 \mathrm{~nm}, 0.3)$ using HR-EBSD and simulated reference patterns. The tetragonalities measured by XRD [16] are indicated by dashed lines. 
Table 1: Summary of Tetragonality Measurements

\begin{tabular}{|l|l|l|l|l|l|}
\hline Sample & Sample & Composition & Predicted & Measured & Measured tetragonality: \\
& $\begin{array}{l}\text { Thickness } \\
(\mathrm{nm})\end{array}$ & Ge at\%) & $\begin{array}{l}\text { tetragonality } \\
\left(10^{-2}\right)\end{array}$ & $\begin{array}{l}\text { (etragonality: real } \\
\text { patterns }{ }^{\mathrm{a}}\left(10^{-2}\right)\end{array}$ & simulated patterns ${ }^{\mathrm{a}}\left(10^{-2}\right)$ \\
\hline$(50 \mathrm{~nm}, 0.2)$ & 47.4 & $19.4 \pm 0.2$ & $1.25 \pm 0.04$ & $1.40 \pm 0.03$ & $1.05 \pm 0.16$ \\
\hline$(35 \mathrm{~nm}, 0.3)$ & 35.9 & $27.9 \pm 0.3$ & $1.93 \pm 0.05$ & $1.90 \pm 0.03$ & $1.91 \pm 0.08$ \\
\hline
\end{tabular}

${ }^{\mathrm{a}}$ Uncertainties represent one standard deviation of the mean value, determined from repeated experimental measurements and propagation of variance when required.

${ }^{b}$ Tetragonality is given in terms of net measured tetragonal distortion between that of the Si and that of the SiGe, as defined in Eq. 14 of [16]. 
Table 1: Summary of Tetragonality Measurements

\begin{tabular}{|l|l|l|l|l|l|}
\hline Sample & Sample & Compositio & Predicted & Measured & Measured tetragonality: \\
Thickness & $\mathrm{n}$ & $\begin{array}{l}\text { tetragonality } \\
\text { (nm) }\end{array}$ & $\begin{array}{l}\text { tetragonality: real } \\
\left(10^{-2}\right)\end{array}$ & $\begin{array}{l}\text { simulated patterns }{ }^{\mathrm{a}}\left(10^{-}\right. \\
\text {patterns }{ }^{\mathrm{a}}\left(10^{-2}\right)\end{array}$ & $\left.{ }^{2}\right)$ \\
\hline$(50 \mathrm{~nm}, 0.2)$ & 47.4 & $19.4 \pm 0.2$ & $1.25 \pm 0.04$ & $1.40 \pm 0.03$ & $1.05 \pm 0.16$ \\
\hline$(35 \mathrm{~nm}, 0.3)$ & 35.9 & $27.9 \pm 0.3$ & $1.93 \pm 0.05$ & $1.90 \pm 0.03$ & $1.91 \pm 0.08$ \\
\hline
\end{tabular}

a Uncertainties represent one standard deviation of the mean value, determined from repeated experimental measurements and propagation of variance when required.

$\mathrm{b}$ Tetragonality is given in terms of net measured tetragonal distortion between that of the Si and that of the SiGe, as defined in Eq. 14 of [16]. 
Click here to download high resolution image

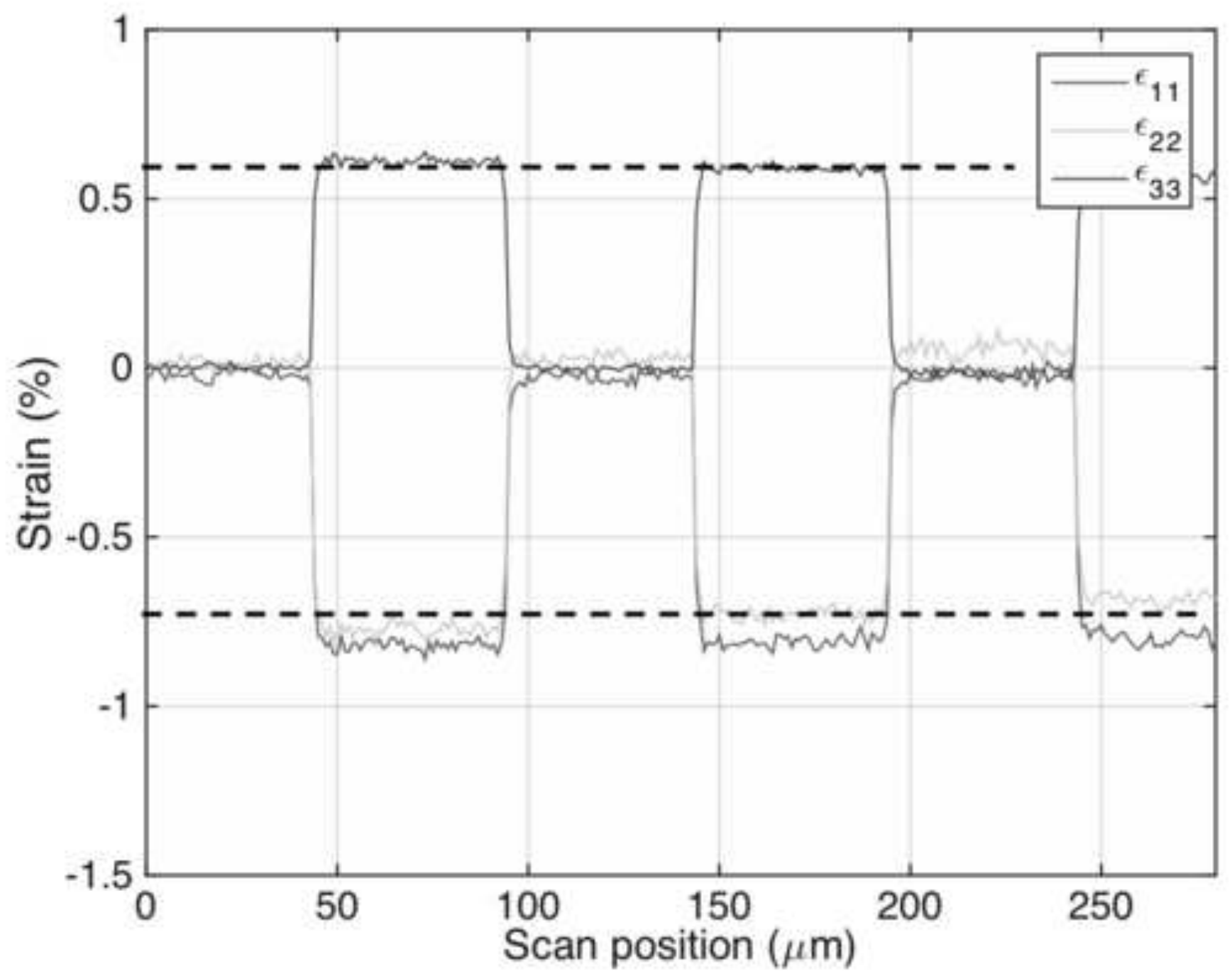


Click here to download high resolution image

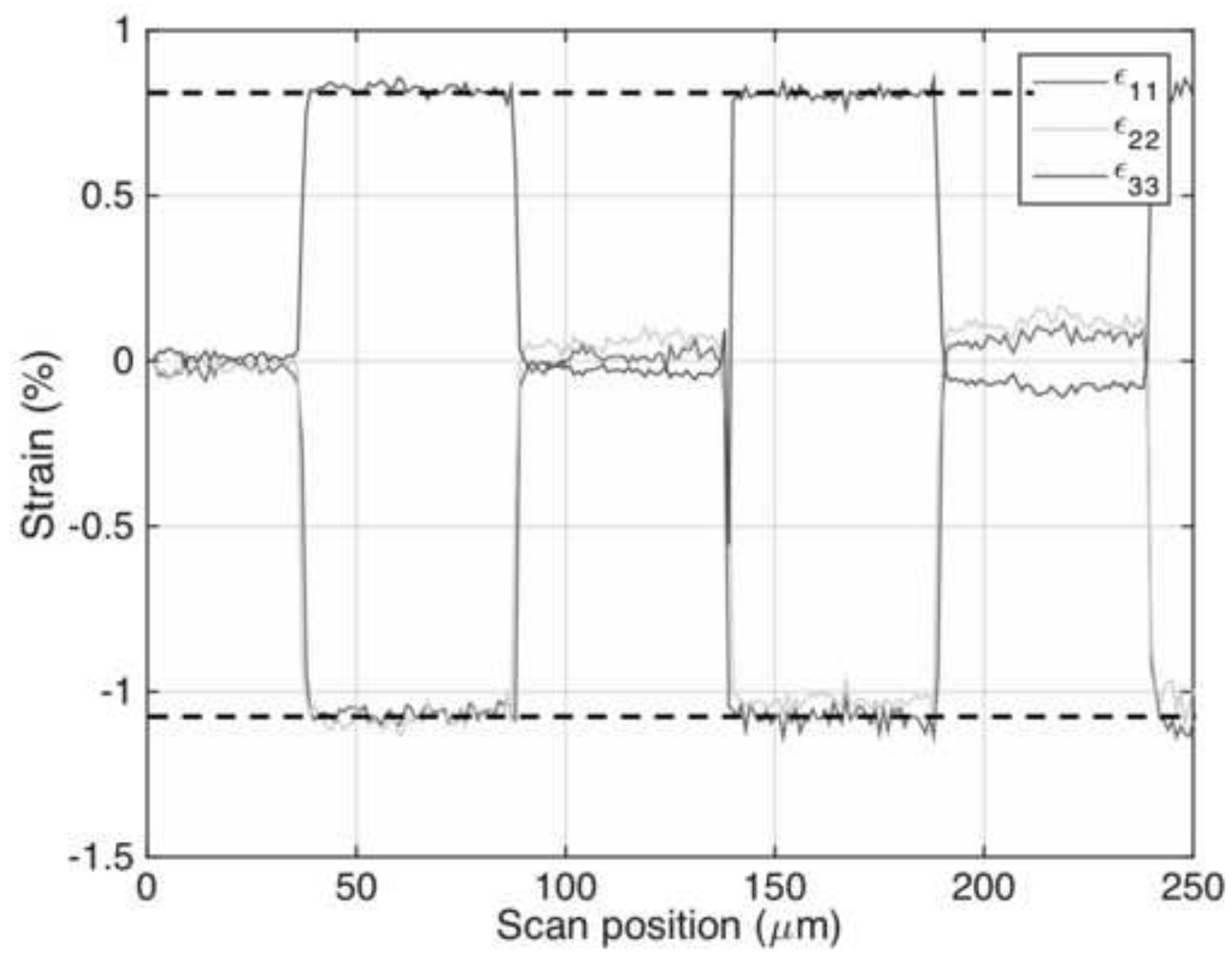




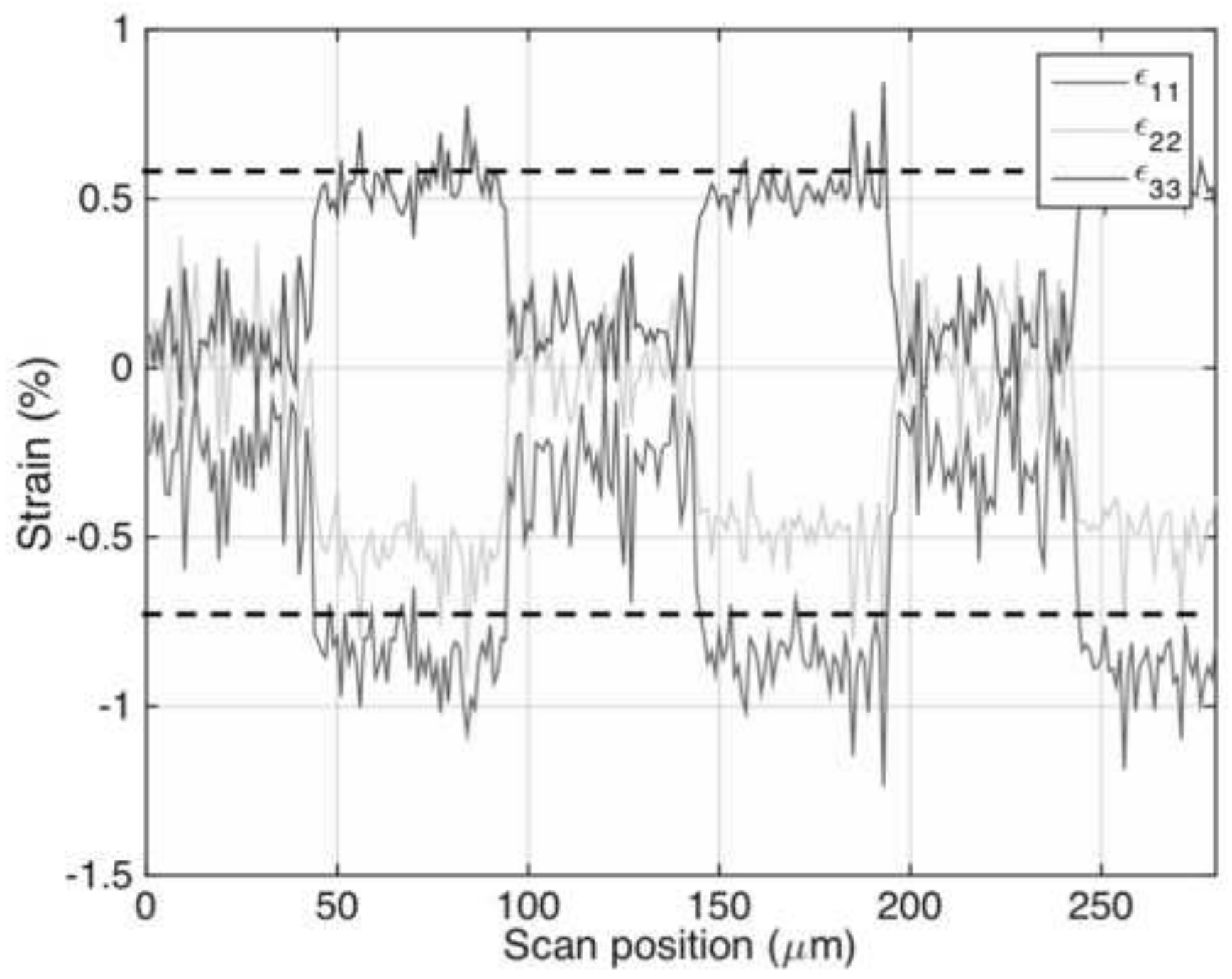




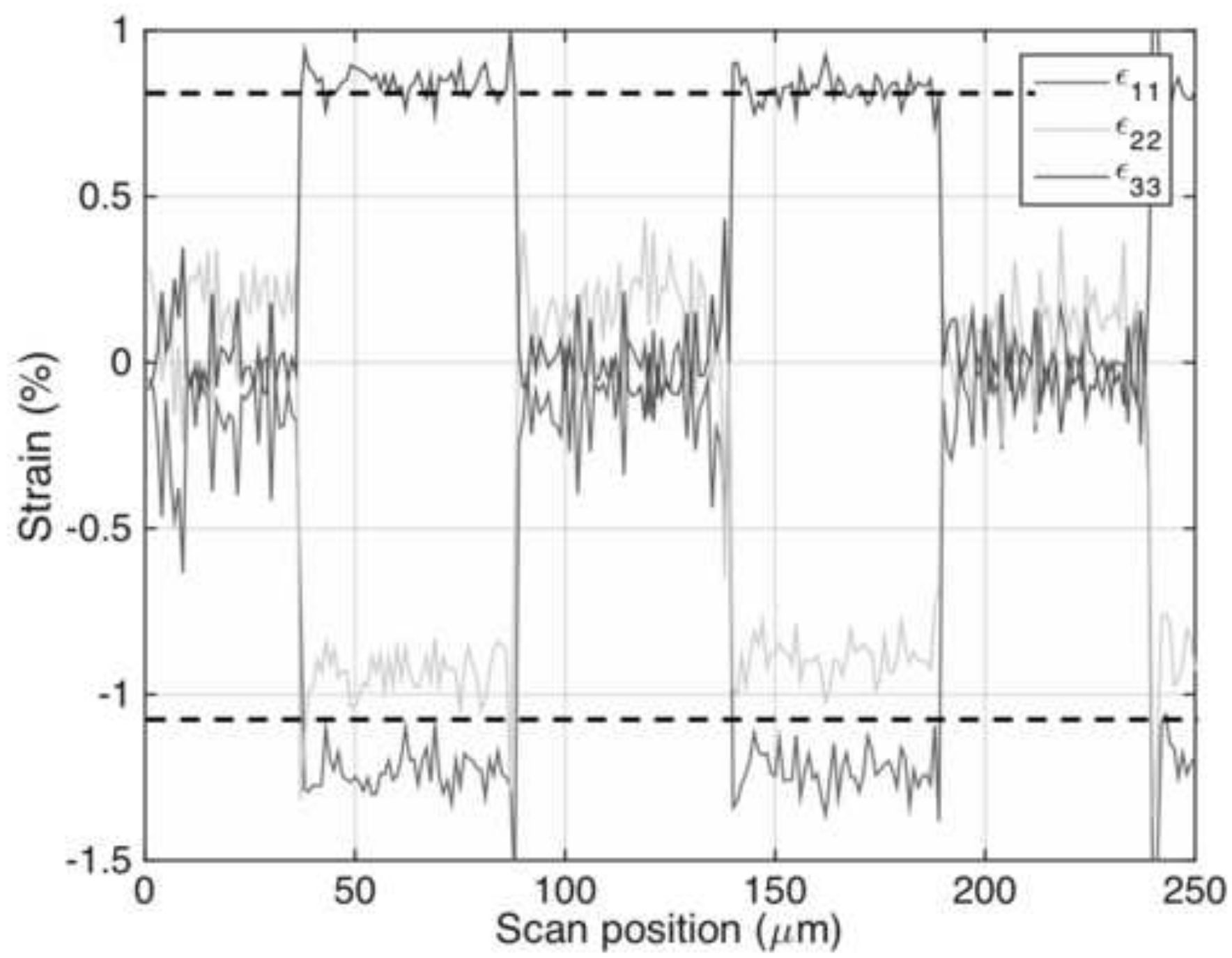


Click here to download high resolution image
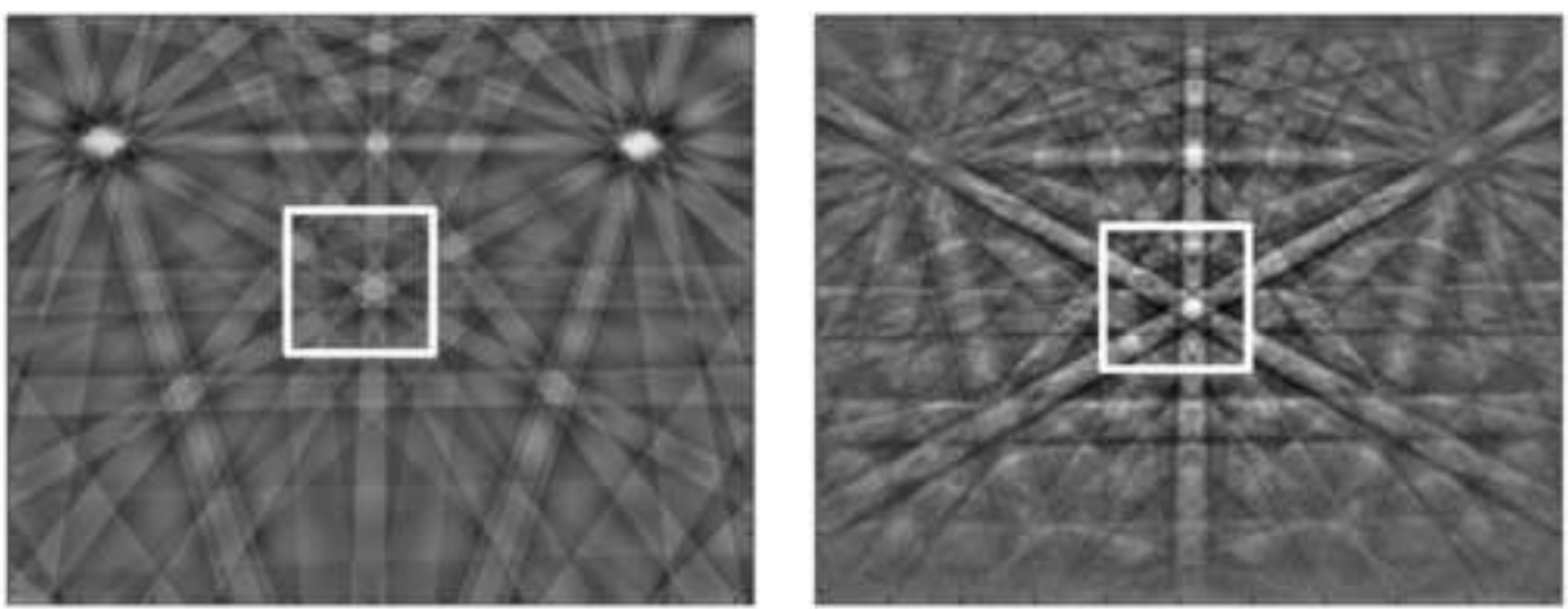


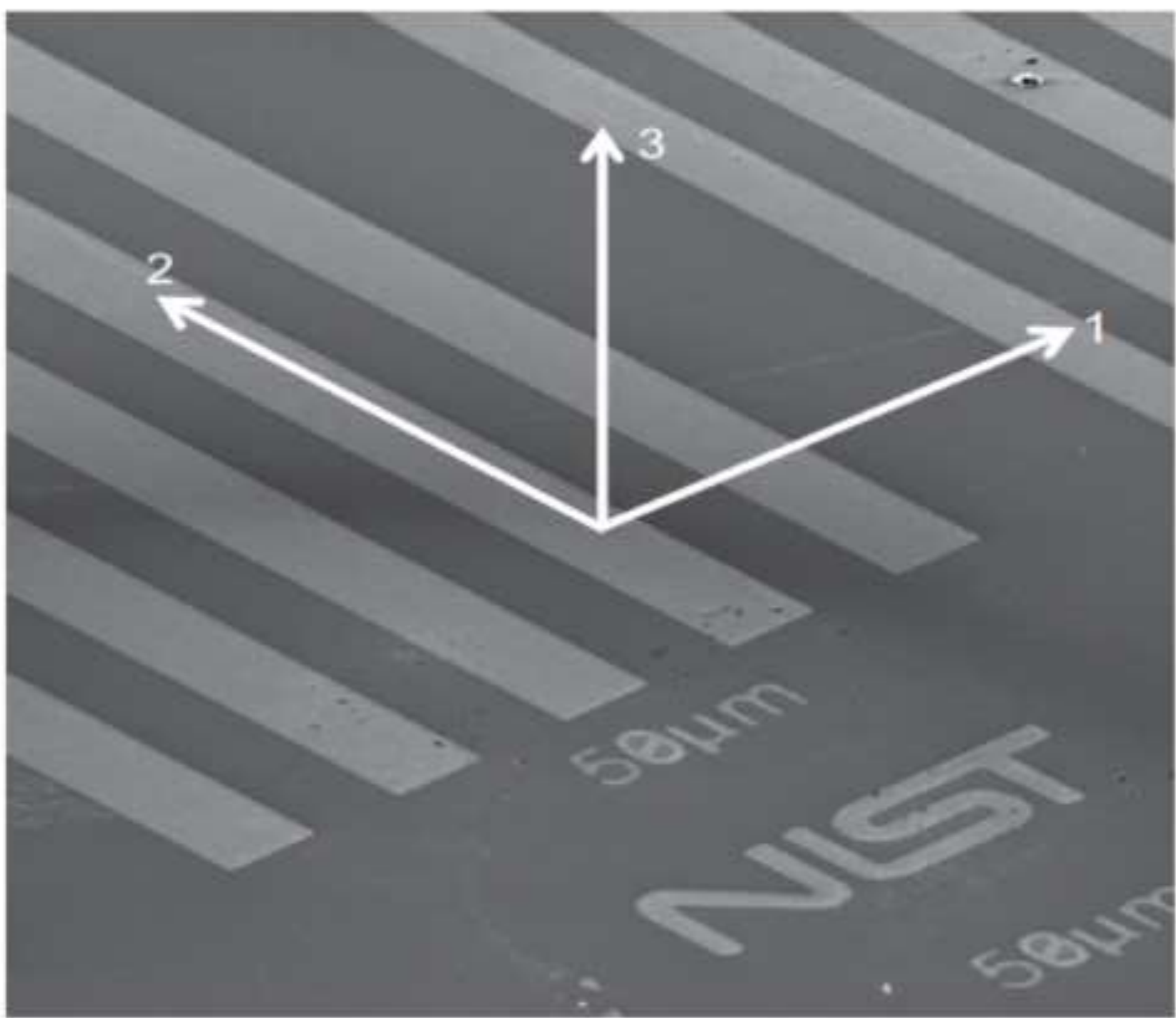




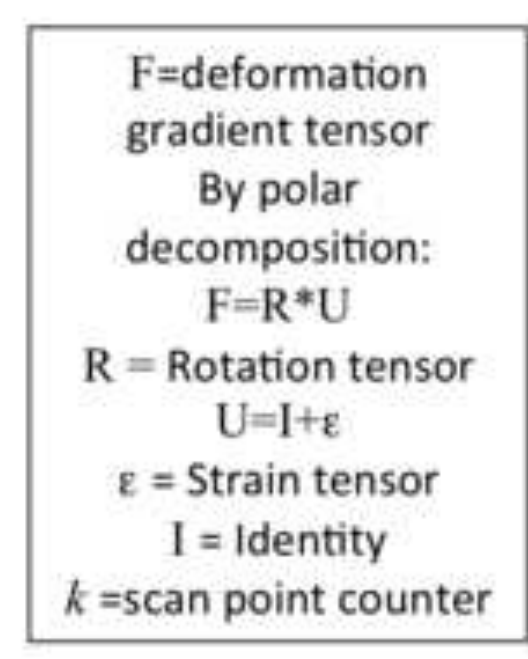

-Adjustable parameters, chosen to give convergence Calculate $\mathrm{g}^{k}=\mathrm{R}_{i}^{\mathrm{T}} \mathrm{g}_{i-1}^{k}$
Set $\mathrm{F}_{i}^{k}=\mathrm{I}$

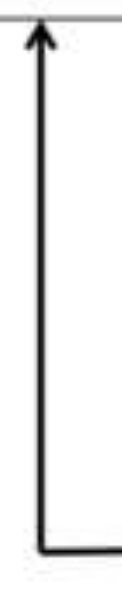

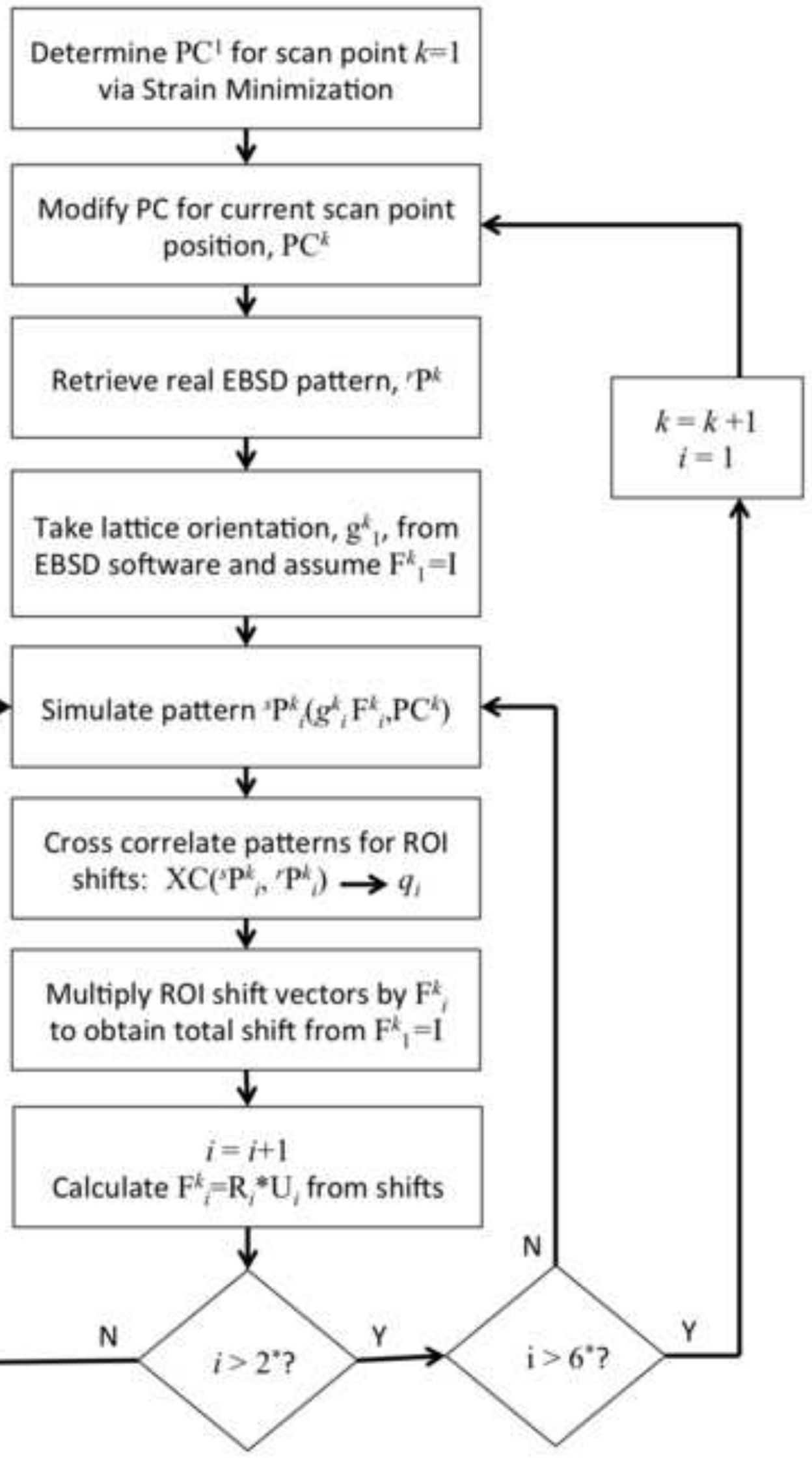

via Strain Minimization position, $\mathrm{PC}^{k}$

$$
\begin{gathered}
k=k+1 \\
t=1
\end{gathered}
$$

Take lattice orientation, $\mathrm{g}_{1}^{k}$, from EBSD software and assume $\mathrm{F}_{1}^{k}=1$

$$
\downarrow
$$

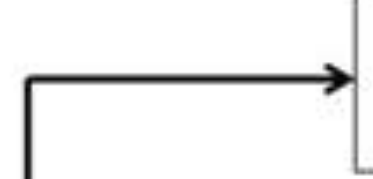

Simulate pattern ${ }^{3} \mathrm{P}_{i}^{k}\left(g_{i}^{k}, \mathrm{~F}_{i}^{k}, \mathrm{PC}^{k}\right)$

\section{$\downarrow$}

ross correlate patterns for ROI shifts: $\mathrm{XC}\left({ }^{s} \mathrm{P}^{k}{ }_{i}, \mathrm{P}^{i}\right) \rightarrow q_{i}$

Multiply ROI shift vectors by $\mathrm{F}^{k}$ to obtain total shift from $\mathrm{F}_{1}^{k}=1$

$$
i=i+1
$$

Calculate $\mathrm{F}_{i}^{k}=\mathrm{R}_{i} * \mathrm{U}_{j}$ from shifts 
Click here to download high resolution image

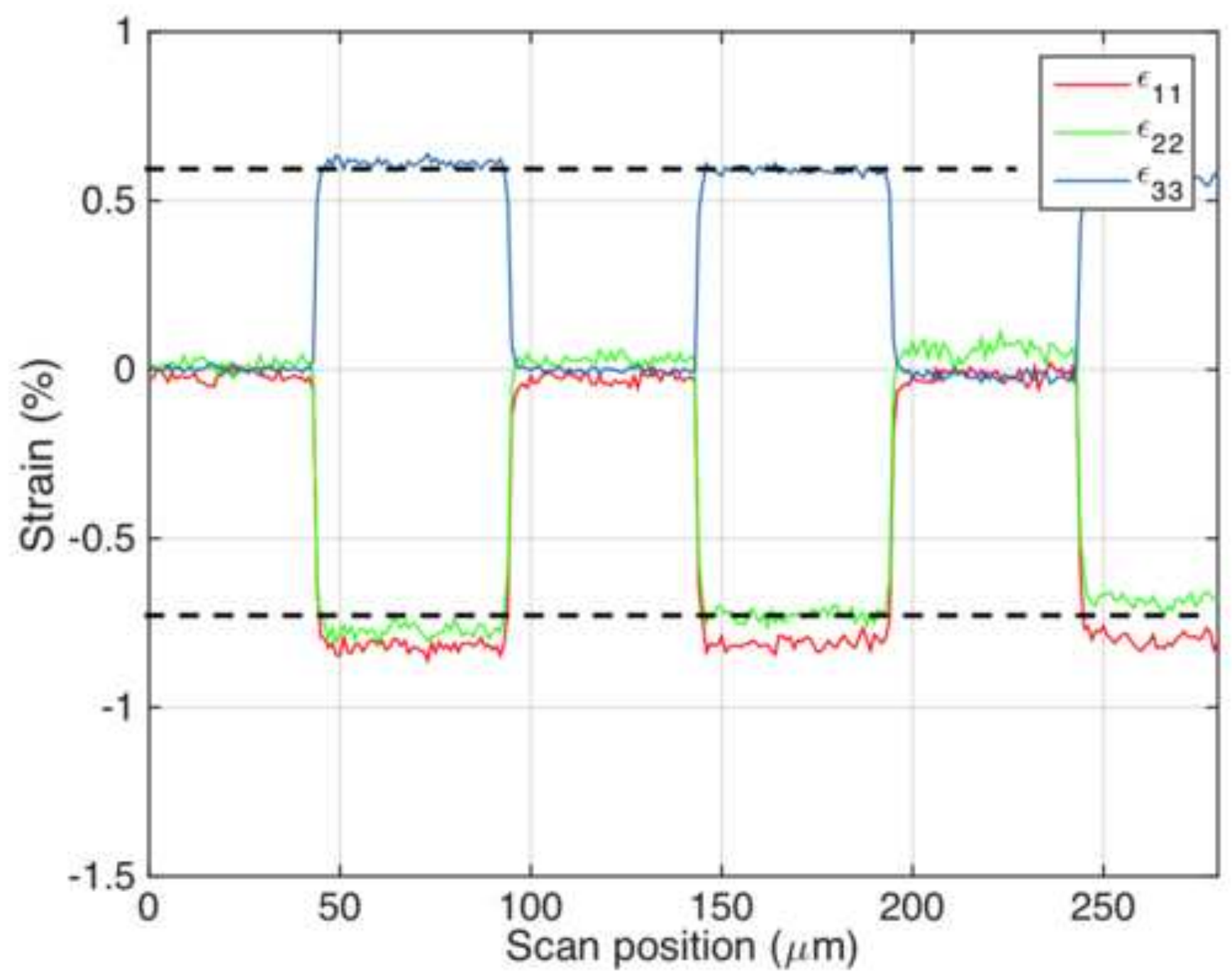


Click here to download high resolution image

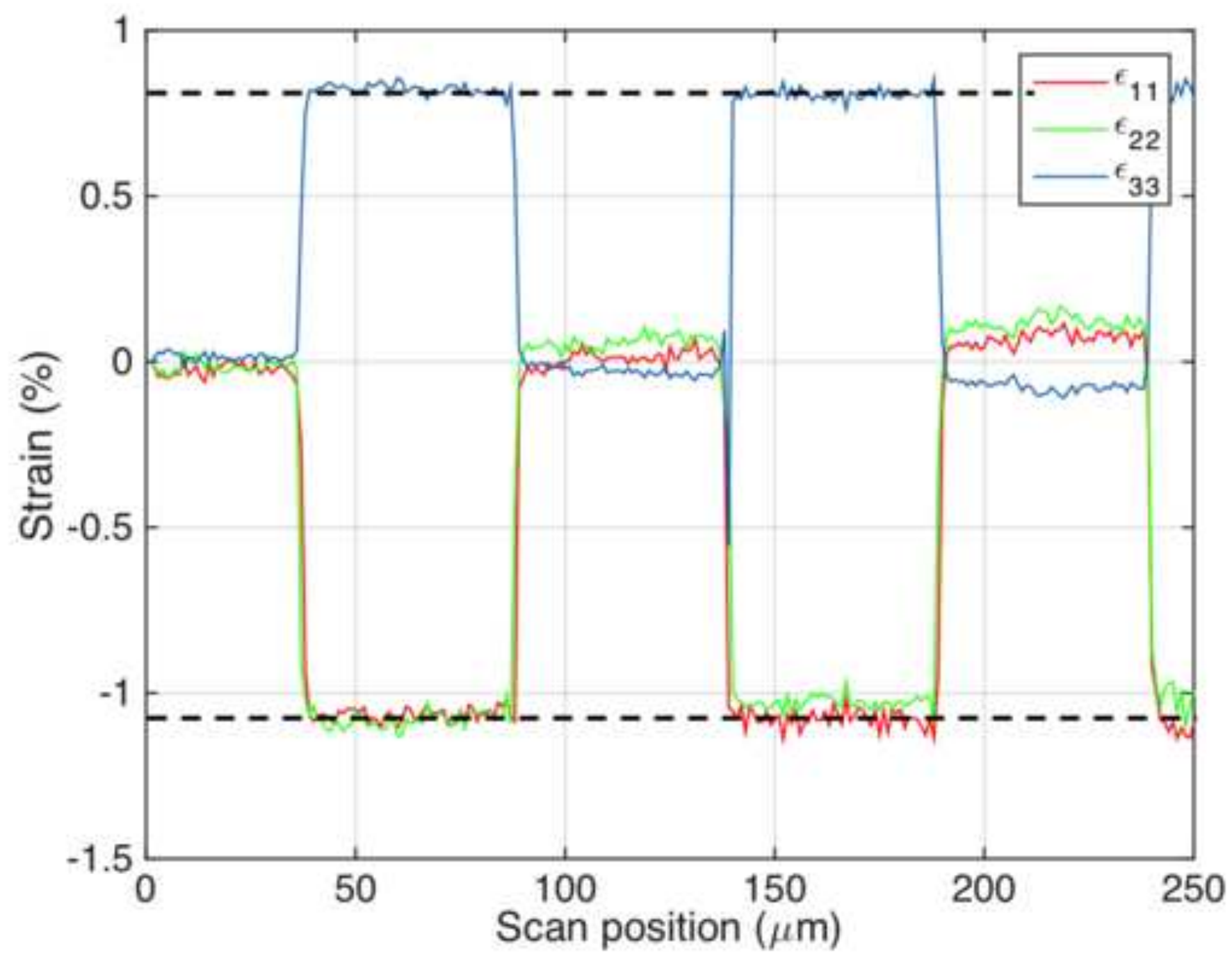




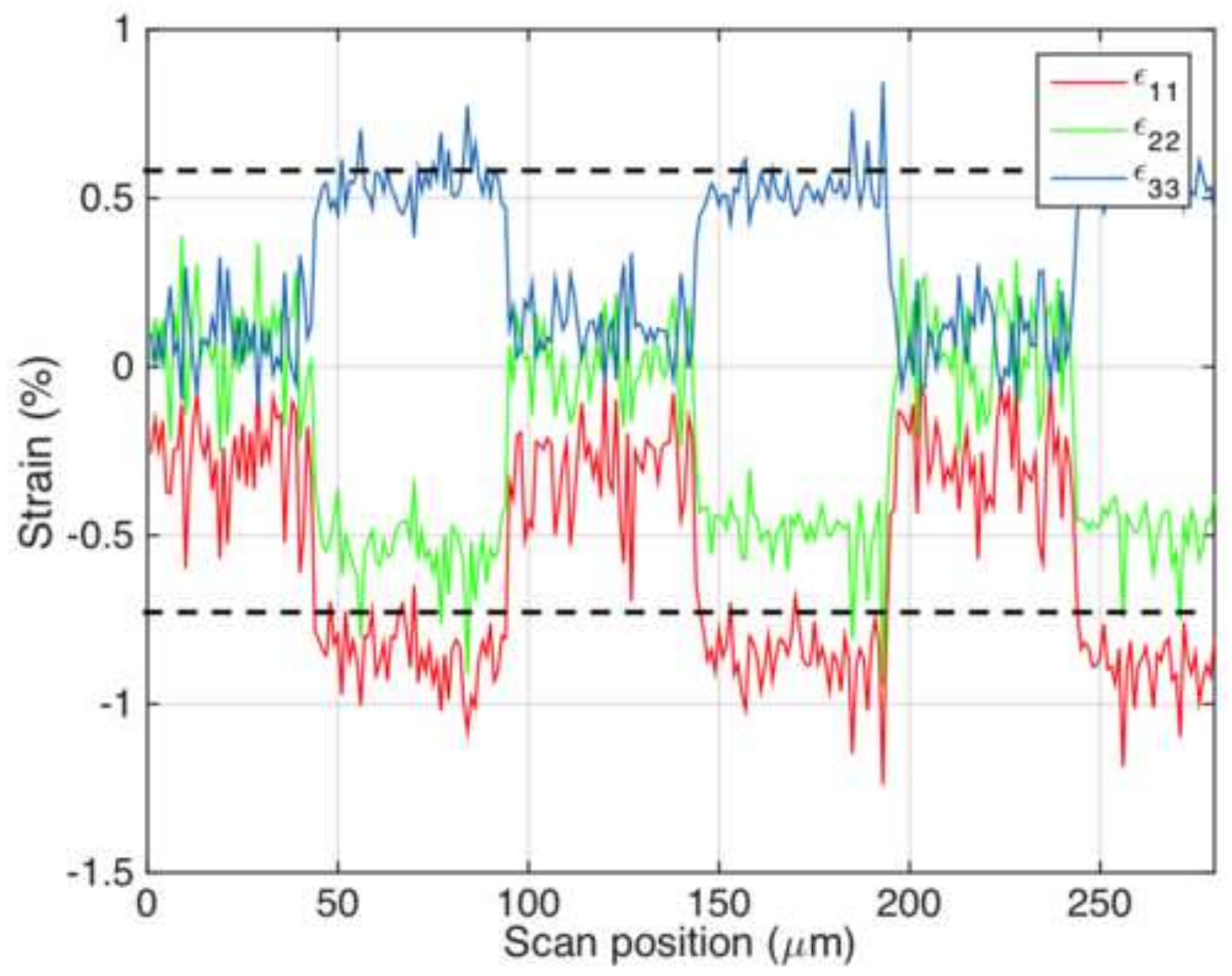




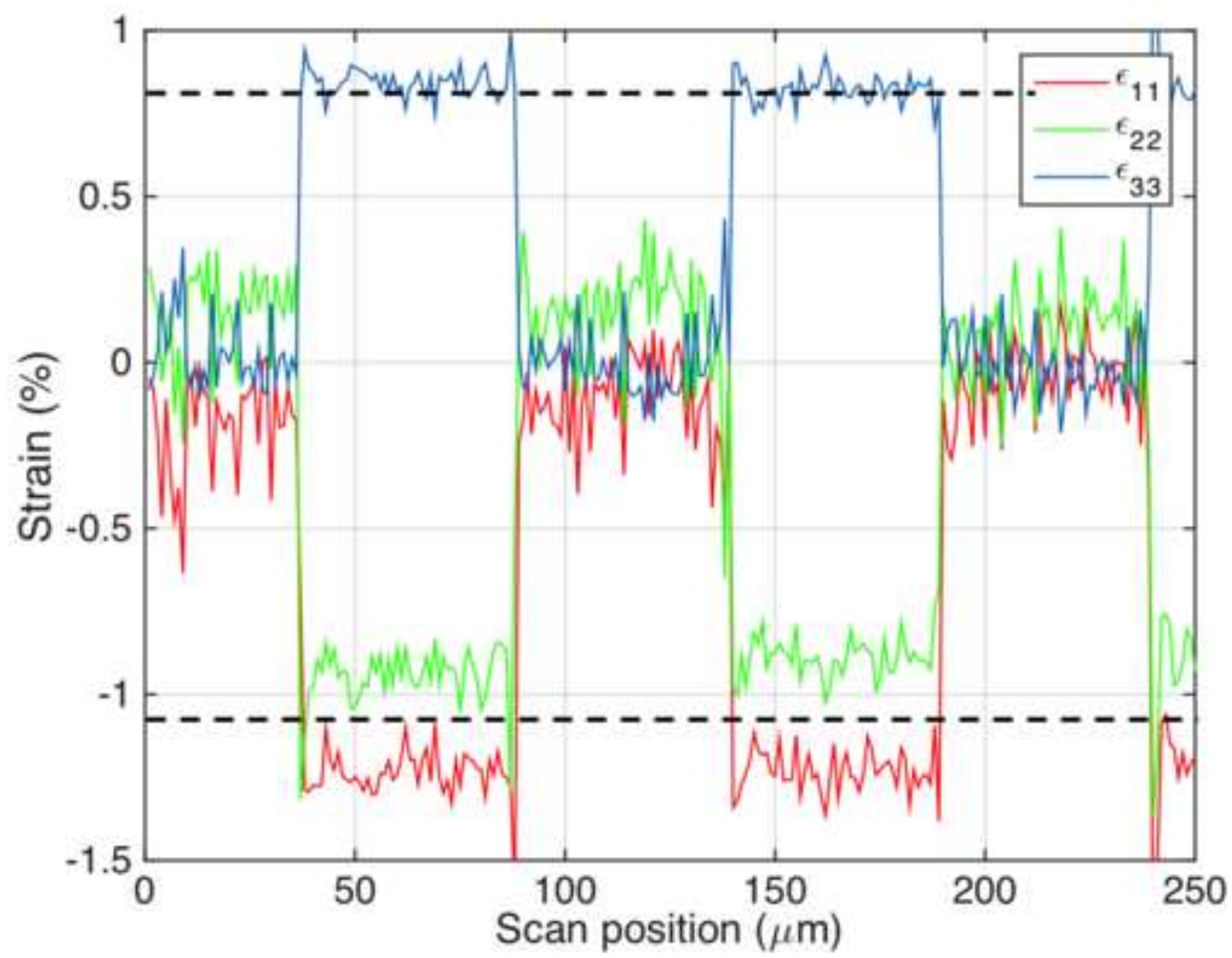


Click here to download high resolution image

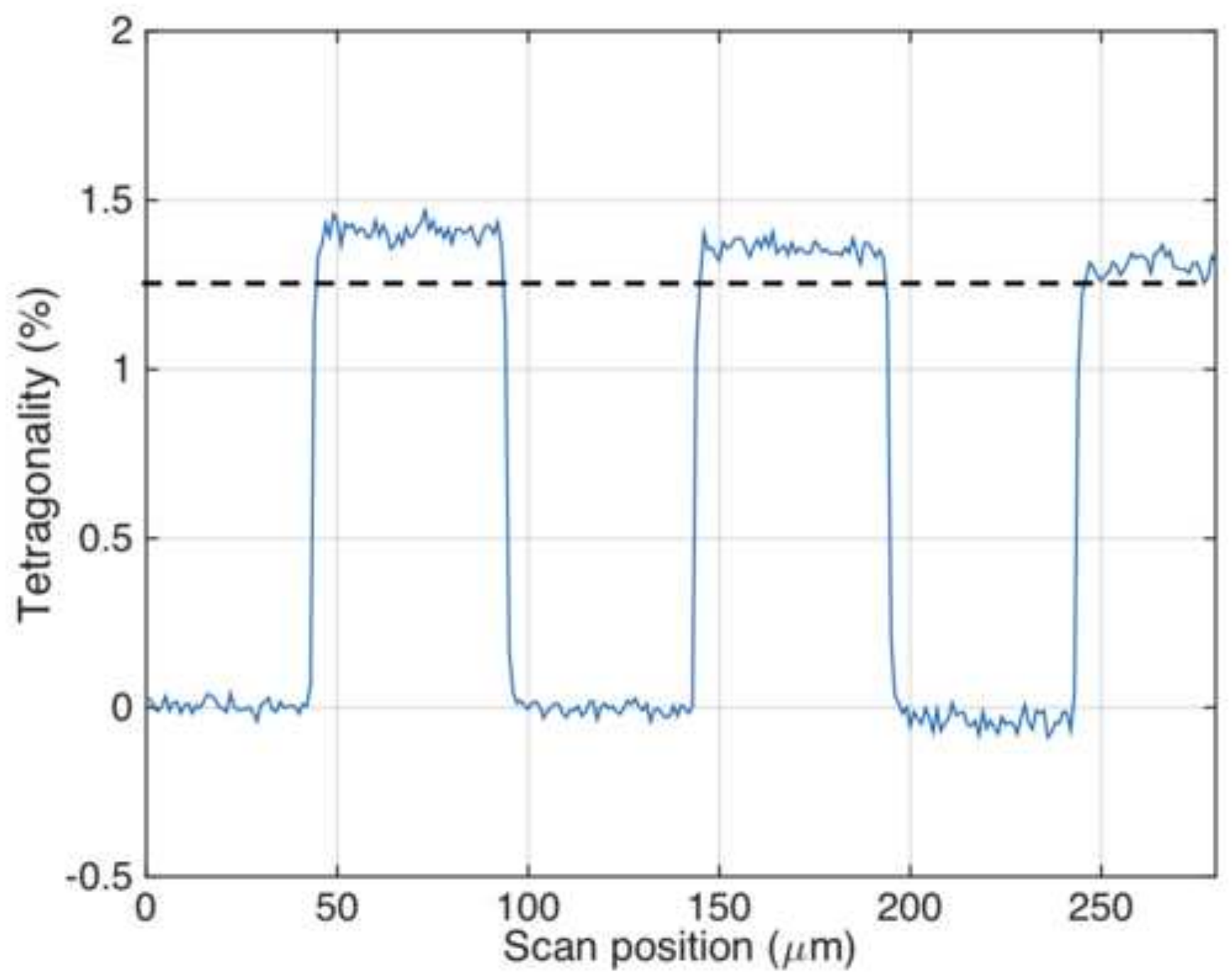


Click here to download high resolution image

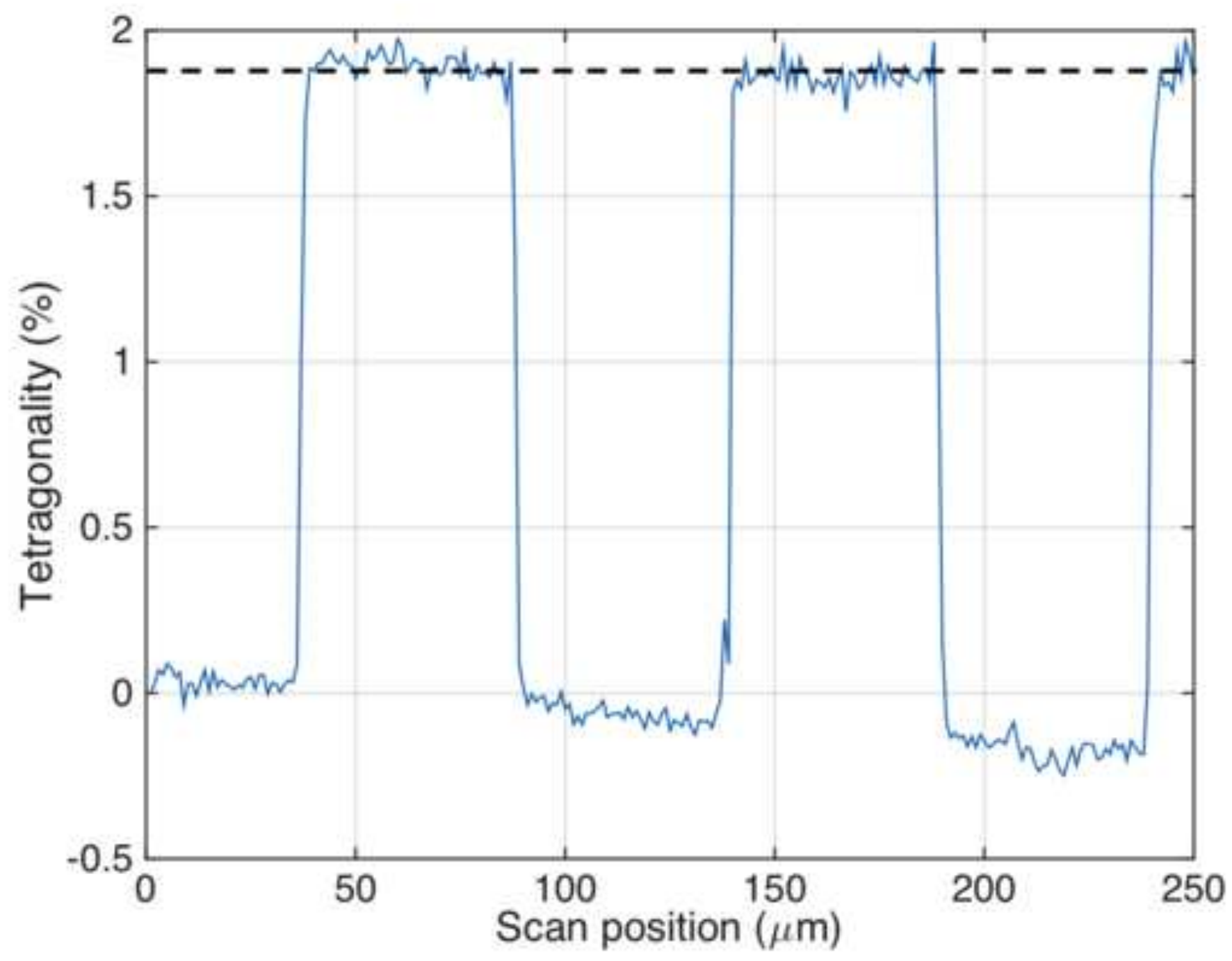




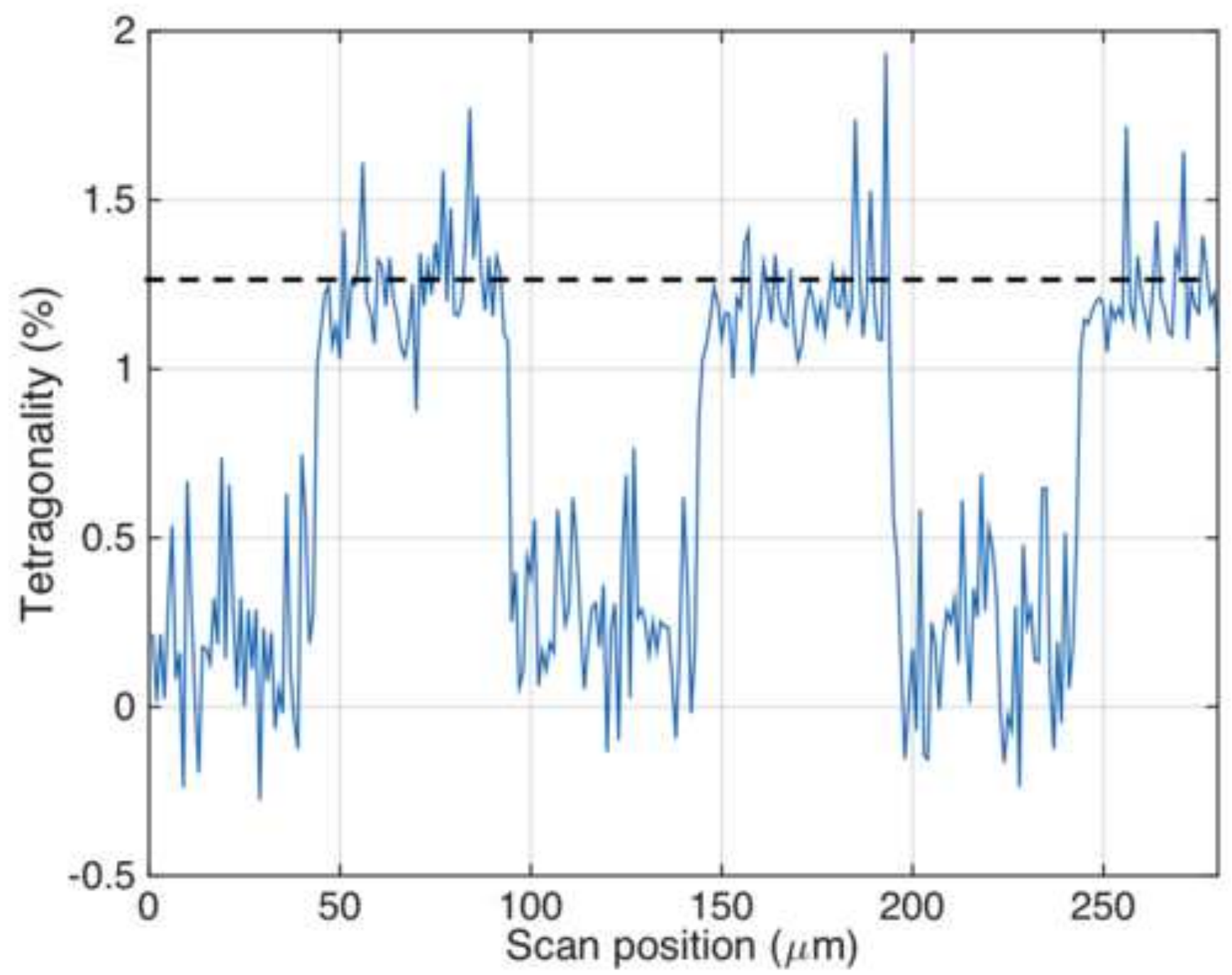


Click here to download high resolution image

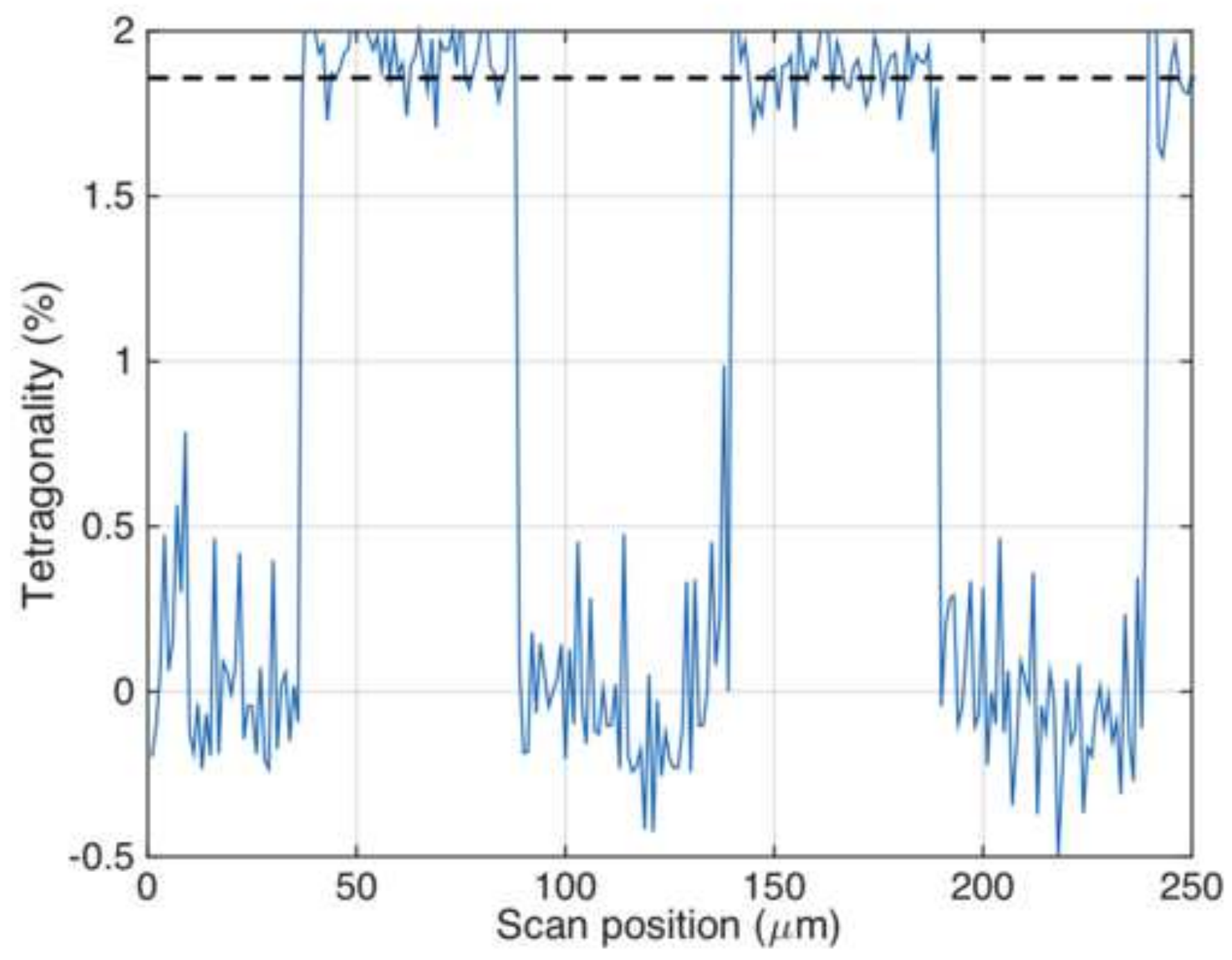

